\title{
Citations
}

\section{Mobile app}

Call it Foursquare for molecules. Just as the popular app gives location information about individual users, a new method reported in Nano Letters can track molecules as they move on or in cells. Described by Benke et al., the technique applies a similar approach to super-resolution imaging. In brief, although the resolution of light microscopy is limited by the wavelength of light, if enough photons can be collected from a discrete source, its location can be determined at an accuracy well beyond the typical limits. While turning on a lone fluorophore is unrealistic, substantial resolution gains can be achieved if activation is limited to a sparse subset. This selectivity can be induced by using a redox environment to drive organic dyes into a metastable dark state from which they stochastically transition to states allowing fluorescence emission. This blinking behavior can optically resolve a given molecule from the wider population. Applying this strategy to tracking single molecules in live cells, however, requires conditions that do not compromise viability. Although the authors tested culture media containing a variety of reducing agents and oxygen scavengers, in most cases Leibovitz medium - a formulation already used for extended live cell imaging - gave adequate blinking for tracking by an algorithm that reconstructs molecular trajectories from sequential frames. In each case, the imaging target was a protein containing a tag for self (SNAP, Halo) or enzyme-mediated (acyl carrier protein $[\mathrm{ACP}])$ labeling. When fused to membrane-bound receptors, three green (Alexa 488, Dy-488, and Oregon Green), two red (TMR and Dy-547), and one far-red (Dy-647) dye all blink well enough for single-molecule tracking. Tracking molecules within intracellular compartments is also possible with cell-permeable
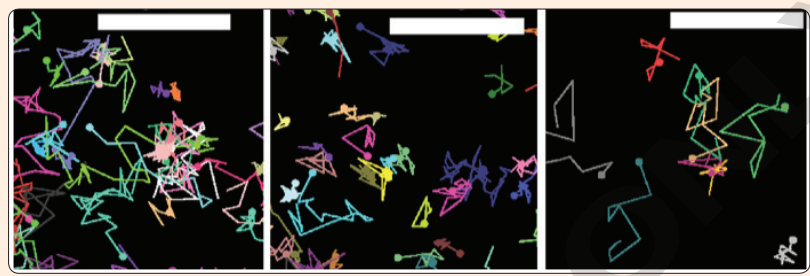

Single-molecule tracks obtained under various buffer conditions. Source: Nano Letters

dyes, as the authors show by monitoring movement of the inner mitochondrial membrane protein Cox $8 \mathrm{~A}$ and the histone $\mathrm{H} 2 \mathrm{~B}$. With the SNAP tag, TMR performed well in mitochondria, while TMR, Oregon Green, and Dy-505 all worked in nuclei. One compartment that could not sustain blinking was the cytosol, at least when tested by tagging cytoskeletal proteins, perhaps because of an unfavorable redox environment. For multicolor imaging, the authors show sequential monitoring of Dy-547-labeled SNAP-tagged $\beta 2$ adrenergic receptor and Alexa 488-labeled Halo-tagged transferrin receptor in cotransfected cells, with subsequent overlay revealing mobility differences of the two receptors. Adding an ACP-tagged protein with a far-red dye should be feasible as well, enabling tracking of 3 proteins in the same cell. As more dyes become available, this nanoscale imaging method will offer even more flexibility for monitoring dynamics of single molecules in living cells.

\section{A. Benke et al. 2012. Multicolor single molecule tracking of stochastically active synthetic dyes. Nano Lett. 12(5):2619-24.}

\section{Identity crisis}

Accurate characterization of stem cells and derived populations is a must-have for understanding stem cell biology and triggering reprogramming. Unfortunately, the field lacks validated cell-surface markers to unambiguously differentiate lineages and permit selective isolation. That's what makes a new proteomics resource from Rugg-Gunn et al., published in Developmental Cell, so exciting. The work focuses on mouse embryonic, epiblast, trophoblast, and extraembryonic endoderm stem cell lineages. Although gene expression analysis might identify markers, the authors were concerned by data suggesting poor correlation in RNA and protein levels among cell-surface proteins. Hence, they utilized a direct proteomics approach, biotinylating cell-surface proteins to prepare affinitypurified samples for mass spectrometry. Hits were screened for proteins whose ontology annotations were consistent with membrane localization and which passed an algorithm trained to detect transmembrane domains. Out of this vetting came 551 proteins. To go from these potential markers to a protein signature indicative of each cell type, Rugg-Gunn et al. used commercially available antibodies to come up with 27 proteins that showed lineage specificity and cell-surface localization by immunofluorescence microscopy. Of these, only two had been previously identified as stem cell markers. Nine antibodies were compatible with flow cytometry, and gave strong cell-type-specific signals. These protein signatures for different stem cell lineages empower several types of studies. First, they can monitor in vitro differentiation and interconversion between pluripotent cell types. Second, many of the markers will be useful for studying early embryo development. Third, the markers make possible sorting of specific cell types from mixed samples in vitro or from dissociated blastocysts; because the sorted cells remain viable, they offer opportunities for functional or genomic studies. Whether approached as a description of reagents useful for isolating and characterizing stem cells or as a data trove of proteins whose role in stem cell biology and development can be elucidated, this new resource from Rugg-Gunn et al. should accelerate research in this already rapidly moving field.

P.J.Rugg-Gunn etal.2012. Cell-surface proteomics identifies lineage-specific markers of embryo-derived stem cells. Dev Cell. 22(4):887-901.

Selected and written by Nijsje Dorman, Ph.D. [ICA

BioTechniques 53:13 (July 2012)

doi 10.2144/000113884

To purchase reprints of this article, contact:

biotechniques@fosterprinting.com 